\title{
Synthesis and Evaluation of Antimitotic Activity of N-Phenyl Tetralones
}

\author{
Umesha Basavaiah $^{1}$, Sowbhagya ${ }^{2}$, Vinaya ${ }^{1}$, Yeriyur Basavaiah Basavaraju ${ }^{1 *}$ \\ ${ }^{1}$ Department of Studies in Chemistry, University of Mysore, Manasagangotri, Mysore - 570 006, Karnataka, India. \\ ${ }^{2}$ Department of Studies in Organic Chemistry, University of Mysore, Manasagangotri, Mysore - 570 006, Karnataka, India.
}

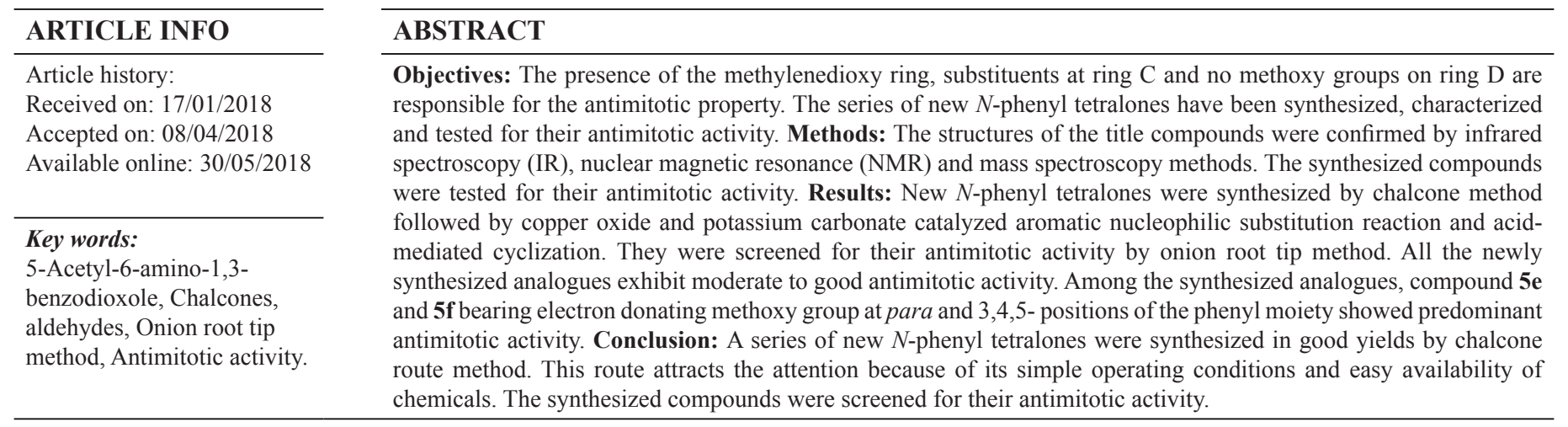

\section{INTRODUCTION}

Among lignan natural products, the aryltetralin lactone podophyllotoxin (1) occupies a unique position. The cytotoxic activity of podophyllotoxin is based on its ability to inhibit the microtubule assembly during cell division. Its use is limited due to side effects when it was used for the treatment of human neoplasia (Shi et al., 2011). The inhibition of the assembly of tubulin into microtubules by the hetero-lignan podophyllotoxin is through tubulin binding, (Damayanthi and Lown, 1998) but high toxicity has limited its therapeutic application making the development of structural analogues having less toxic and water-soluble (Hande, 1998).

Podophyllotoxin and its synthetic derivatives include etoposide, teniposide, and etopophos which display a wide range of medical applications such as vesicant, purgative, antirheumatic,

\section{${ }^{*}$ Corresponding Author}

Yeriyur Basavaiah Basavaraju, Department of Studies in Chemistry, University of Mysore, Manasagangotri, Mysore-570 006, Karnataka, India.E-mail: basavaraju_yb@yahoo.co.in antitumor (Jackson and Dewick, 1985), anti AIDS, antimalarial, cathartic, cytotoxic, fungicidal activities (Lee et al., 1997; Rivera et al., 1975; Gordaliza et al., 2000). It has already been known that podophyllotoxin analogues showed antineoplastic and antiviral properties and its numerous semi-synthetic derivatives have been developed as effective antineoplastic drugs in the field of medical research (Liu et al., 2008).

In recent years many scientists focused on the development of podophyllotoxin analogues possessing high biological activity, low toxicity, and safety towards environmentfriendly behavior. Therefore, it was decided to synthesize podophyllotoxin analogues by modifying ring $\mathrm{C}$ in the podophyllotoxin skeleton. Hence the introduction of nitrogen in ring $\mathrm{C}$ of podophyllotoxin might exhibit more antimitotic activity compared to podophyllotoxin and its derivatives.

\section{EXPERIMENTAL SECTION}

\section{Materials and methods}

All the chemicals of the analytical grade used in 
this work were purchased from $\mathrm{CDH}$ chemicals and used as provided directly unless otherwise stated. The melting points were measured by electrothermal apparatus and are uncorrected. The IR spectra were recorded on an FT-IR instrument in $\mathrm{KBr}$ disc. The ${ }^{1} \mathrm{H}$ NMR (400 MHz) spectra and ${ }^{13} \mathrm{C}$ NMR (100 MHz) spectra were recorded on Agilent 400MR DD2 spectrometer using $\mathrm{CDCl}_{3}$ as a solvent (chemical shift in $\delta \mathrm{ppm}$ ), using TMS as an internal standard. The mass spectra were performed using
Waters, USA on Synapt G2 HDMS/ACQUITY UPLC instrument. The elemental analysis was recorded on a Perkin-Elmer 2400 instrument. Following up the reactions and checking the purity of the compounds were performed by means of TLC in benzene and ethyl acetate mixture (7:0.5). The synthesized compounds (5a-h) were purified by column chromatography using silica gel (60-120 mesh) as adsorbent and benzene as eluent.
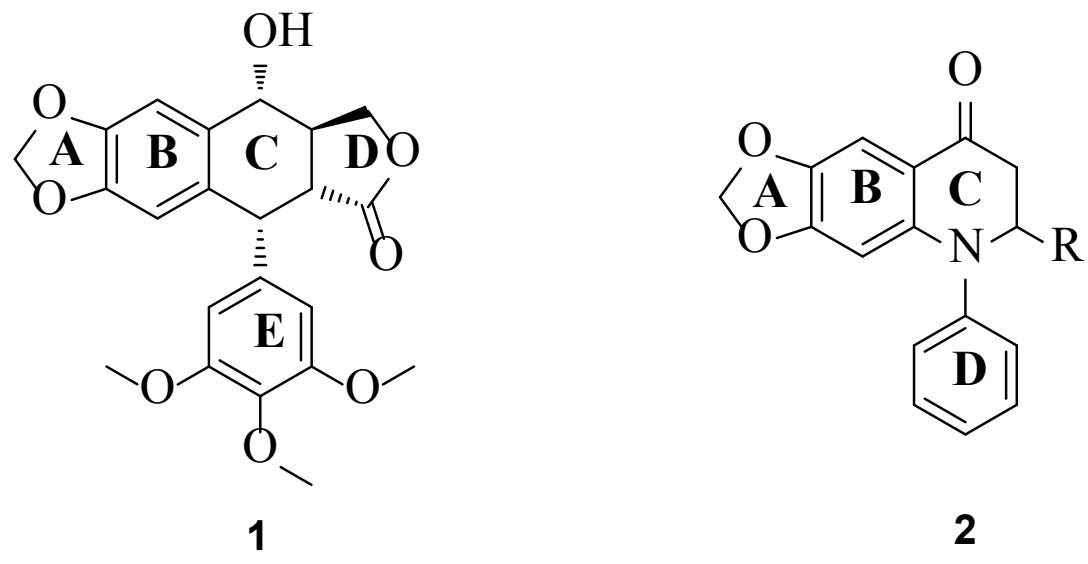

Fig. 1: Structure of podophyllotoxin (1) and newly synthesized $N$-phenyl tetralones (2).

\section{General procedure for the preparation of chalcones (3a-h)}

5-Acetyl-6-amino-1,3-benzodioxole (1) (1.79 g, 10 $\mathrm{mmol})$ and substituted benzaldehydes (2a-h) $(10 \mathrm{mmol})$ were stirred vigorously in $60 \mathrm{~mL}$ of $30 \%$ methanolic $\mathrm{NaOH}(0.8 \mathrm{~g}, 20$ $\mathrm{mmol})$ in the presence of sodium hydroxide at $15-30^{\circ} \mathrm{C}$ for $4 \mathrm{hrs}$ then the reaction mixture was kept overnight in an ice bath. The precipitated products (3a-h) were filtered off and recrystallized from ethanol.

1-(6-Aminobenzo[d][1,3]dioxol-5-yl)-3-phenylprop-2-en-1-one (3a)

Colour: yellow solid. Yield: $72.0 \%$. M.p.: $97-99^{\circ} \mathrm{C}$. IR $\left(\mathrm{KBr}, v, \mathrm{~cm}^{-1}\right): 1665(\mathrm{C}=\mathrm{O}), 1592(\mathrm{C}=\mathrm{C}) .{ }^{1} \mathrm{H}$ NMR $(400 \mathrm{MHz}$, $\mathrm{CDCl}_{3}, \delta, \mathrm{ppm}$ ): 8.08 (d, $\left.1 \mathrm{H}, J=14 \mathrm{~Hz}, \beta-\mathrm{CH}\right), 7.65$ (s, 1H, H-4'), 7.27 (s, 1H, H-1'), 7.04 (d, 1H, $J=14$ Hz, $\alpha-\mathrm{CH}), 6.89-6.57$ (m, 5H, H-2", H-3", H-4", H-5", H-6"), 6.02 (s, 2H, OCH $\mathrm{O}$ ), 4.06 $\left(\mathrm{s}, 2 \mathrm{H}, \mathrm{NH}_{2}\right) .{ }^{13} \mathrm{C} \mathrm{NMR}\left(100 \mathrm{MHz}, \mathrm{CDCl}_{3}, \delta, \mathrm{ppm}\right): 189.7,153.9$, $148.8,145.1,144.1,135.2,128.6,128.5,127.9,123.2,121.3$, 103.6, 101.2, 100.9. MS (ESI) m/z: $267.02\left(M^{+}\right)$. Anal. Calcd. for $\mathrm{C}_{16} \mathrm{H}_{13} \mathrm{NO}_{3}$ : C, 71.90; H, 4.90; N, 5.24. Found: C, 71.87; H, 4.95; $\mathrm{N}, 5.21 \%$.

1-(6-Aminobenzo[d][1,3]dioxol-5-yl)-3-(4-chlorophenyl)prop-2en-1-one (3b)

Colour: yellow solid. Yield: $65.0 \%$. M.p.: $110-112^{\circ} \mathrm{C}$. IR $\left(\mathrm{KBr}, v, \mathrm{~cm}^{-1}\right): 1666(\mathrm{C}=\mathrm{O}), 1586(\mathrm{C}=\mathrm{C}) .{ }^{1} \mathrm{H}$ NMR $(400 \mathrm{MHz}$, $\left.\mathrm{CDCl}_{3}, \delta, \mathrm{ppm}\right): 7.96$ (d, $\left.1 \mathrm{H}, J=12 \mathrm{~Hz}, \beta-\mathrm{CH}\right), 7.33$ (s, 1H, H-4'), 7.10 (s, 1H, H-1'), 7.26 (d, 1H, $J=12 \mathrm{~Hz}, \alpha-\mathrm{CH}), 7.02-6.72$ (m, 4H, H-2", H-3", H-5", H-6"), 6.16 (s, 2H, OCH $\mathrm{O}$ ), 4.10 (s, $\left.2 \mathrm{H}, \mathrm{NH}_{2}\right) .{ }^{13} \mathrm{C} \mathrm{NMR}\left(100 \mathrm{MHz}, \mathrm{CDCl}_{3}, \delta, \mathrm{ppm}\right): 188.5,152.4$, $148.1,144.5,140.1,133.8,133.0,129.3,128.1,123.5,120.9$, 101.3, 100.1, 100.0. MS (ESI) m/z: $301.01\left(M^{+}\right)$. Anal. Calcd. for $\mathrm{C}_{16} \mathrm{H}_{12} \mathrm{ClNO}_{3}$ : C, 63.69; H, 4.01; N, 4.64. Found: C, 63.65; H, $4.04 ; \mathrm{N}, 4.63 \%$.

1-(6-Aminobenzo[d][1,3]dioxol-5-yl)-3-(4-fluorophenyl)prop-2en-1-one (3c)

Colour: yellow solid. Yield: $62.0 \%$. M.p.: $92-94{ }^{\circ} \mathrm{C}$. IR $\left(\mathrm{KBr}, v, \mathrm{~cm}^{-1}\right): 1669(\mathrm{C}=\mathrm{O}), 1592(\mathrm{C}=\mathrm{C}) .{ }^{1} \mathrm{H}$ NMR $(400 \mathrm{MHz}$, $\left.\mathrm{CDCl}_{3}, \delta, \mathrm{ppm}\right): 8.02$ (d, $\left.1 \mathrm{H}, J=13 \mathrm{~Hz}, \beta-\mathrm{CH}\right), 7.30-7.18(\mathrm{~m}, 4 \mathrm{H}$, H-2", H-3", H-5", H-6"), 7.22 (s, 1H, H-4'), 6.76 (s, 1H, H-1'), 6.90 (d, $1 \mathrm{H}, J=13 \mathrm{~Hz}, \alpha-\mathrm{CH}), 6.03$ (s, 2H, $\left.\mathrm{OCH}_{2} \mathrm{O}\right), 4.05$ (s, $\left.2 \mathrm{H}, \mathrm{NH}_{2}\right) \cdot{ }^{13} \mathrm{C} \mathrm{NMR}\left(100 \mathrm{MHz}, \mathrm{CDCl}_{3}, \delta, \mathrm{ppm}\right): 186.4,162.8$, $153.5,148.1,145.6,144.3,130.4,130.0,123.8,121.0,114.9$, 103.3, 101.7, 100.0. MS (ESI) m/z: $285.00\left(M^{+}\right)$. Anal. Calcd. for $\mathrm{C}_{16} \mathrm{H}_{12} \mathrm{FNO}_{3}$ : C, 67.36; H, 4.24; N, 4.91. Found: C, 67.32; H, 4.26; $\mathrm{N}, 4.90 \%$.

1-(6-aminobenzo[d][1,3]dioxol-5-yl)-3-(4-nitrophenyl)prop-2en-1-one (3d)

Colour: dark yellow solid. Yield: 55.0\%. M.p.: 112$114^{\circ} \mathrm{C}$. IR $\left(\mathrm{KBr}, v, \mathrm{~cm}^{-1}\right): 1665(\mathrm{C}=\mathrm{O}), 1588(\mathrm{C}=\mathrm{C}) .{ }^{1} \mathrm{H}$ NMR $(400$ $\left.\mathrm{MHz}, \mathrm{CDCl}_{3}, \delta, \mathrm{ppm}\right): 7.82(\mathrm{~d}, 1 \mathrm{H}, J=15 \mathrm{~Hz}, \beta-\mathrm{CH}), 7.68-7.72$ (m, 4H, H-2", H-3", H-5", H-6"), 7.21 (s, 1H, H-4'), 7.08 (s, 1H, H-1'), 6.83 (d, 1H, J=15 Hz, $\alpha-\mathrm{CH}), 6.07$ (s, 2H, $\left.\mathrm{OCH}_{2} \mathrm{O}\right), 4.13$ (s, $\left.2 \mathrm{H}, \mathrm{NH}_{2}\right) .{ }^{13} \mathrm{C} \mathrm{NMR}\left(100 \mathrm{MHz}, \mathrm{CDCl}_{3}, \delta, \mathrm{ppm}\right): 181.0,153.9$, $151.6,148.0,145.9,144.5,130.1,127.5,123.4,121.9,115.4$, 103.3, 101.0, 100.1. MS (ESI) m/z: $328.01\left(M^{+}\right)$. Anal. Calcd. for $\mathrm{C}_{16} \mathrm{H}_{12} \mathrm{~N}_{2} \mathrm{O}_{6}$ : C, 58.54; H, 3.68; N, 8.53. Found: C, 58.51; H, 3.71; $\mathrm{N}, 8.52 \%$.

1-(6-Aminobenzo[d][1,3]dioxol-5-yl)-3-(4-methoxyphenyl)prop2-en-1-one (3e)

Colour: yellow solid. Yield: $82.0 \%$. M.p.: $104-106^{\circ} \mathrm{C}$. 
IR $\left(\mathrm{KBr}, v, \mathrm{~cm}^{-1}\right): 1665(\mathrm{C}=\mathrm{O}), 1584(\mathrm{C}=\mathrm{C}) .{ }^{1} \mathrm{H}$ NMR $(400 \mathrm{MHz}$, $\left.\mathrm{CDCl}_{3}, \delta, \mathrm{ppm}\right): 8.01$ (d, $\left.1 \mathrm{H}, J=12 \mathrm{~Hz}, \beta-\mathrm{CH}\right), 7.29$ (s, 1H, H-4'), 7.16 (s, 1H, H-1'), 7.03 (d, 1H, J=12 Hz, $\alpha-\mathrm{CH}), 6.84-6.68$ (m, 4H, H-2", H-3", H-5", H-6"), 6.12 (s, 2H, $\left.\mathrm{OCH}_{2} \mathrm{O}\right), 3.96$ (s, 2H, $\left.\mathrm{NH}_{2}\right), 3.80\left(\mathrm{~s}, 3 \mathrm{H}, \mathrm{OCH}_{3}\right) .{ }^{13} \mathrm{C} \mathrm{NMR}\left(100 \mathrm{MHz} \mathrm{CDCl}_{3}, \delta, \mathrm{ppm}\right)$ : $187.2,159.0,153.3,148.4,145.2,144.5,130.4,127.1,124.0$, 121.9, 114.2, 103.2, 101.5, 100.3, 55.1. MS (ESI) m/z: 297.16 $\left(M^{+}\right)$. Anal. Calcd. for $\mathrm{C}_{17} \mathrm{H}_{15} \mathrm{NO}_{4}$ : C, 68.68; H, 5.09; N, 4.71. Found: C, 68.71; H, 5.05; N, 4.73\%.

1-(6-Aminobenzo[d][1,3]dioxol-5-yl)-3-(3,4,5-trimethoxyphenyl) prop-2-en-1-one (3f)

Colour: yellow solid. Yield: $76.0 \%$. M.p.: $121-123^{\circ} \mathrm{C}$. IR ( $\left.\mathrm{KBr}, v, \mathrm{~cm}^{-1}\right): 1663(\mathrm{C}=\mathrm{O}), 1592(\mathrm{C}=\mathrm{C}) .{ }^{1} \mathrm{H}$ NMR $(400 \mathrm{MHz}$, $\left.\mathrm{CDCl}_{3}, \delta, \mathrm{ppm}\right): 7.90(\mathrm{~d}, 1 \mathrm{H}, J=13 \mathrm{~Hz}, \beta-\mathrm{CH}), 7.23$ (d, $1 \mathrm{H}, J=$ $13 \mathrm{~Hz}, \alpha-\mathrm{CH}), 7.18$ (s, 1H, H-4'), 7.12 (s, 1H, H-1'), 6.78 (s, 2H, H-2", H-6"), 6.11 (s, 2H, $\left.\mathrm{OCH}_{2} \mathrm{O}\right), 4.02$ (s, 2H, NH ), 3.86 (s, 9H, $\left.\mathrm{OCH}_{3}\right) \cdot{ }^{13} \mathrm{C} \mathrm{NMR}\left(100 \mathrm{MHz}, \mathrm{CDCl}_{3}, \delta\right.$, ppm): 186.2, 155.1, 153.4, 147.4, 146.3, 144.6, 138.0, 126.9, 123.8, 121.0, 104.3, 103.0, 101.8, 100.5, 60.3, 56.4. MS (ESI) m/z: $357.15\left(M^{+}\right)$. Anal. Calcd. for $\mathrm{C}_{19} \mathrm{H}_{19} \mathrm{NO}_{6}$ : C, 63.86; H, 5.36; N, 3.92. Found: C, 63.88; H, $5.34 ; \mathrm{N}, 3.91 \%$.

1-(6-aminobenzo[d][1,3]dioxol-5-yl)-3-(p-tolyl)prop-2-en-1-one (3g)

Colour: light yellow solid. Yield: $73.0 \%$. M.p.: $97-99^{\circ} \mathrm{C}$. IR $\left(\mathrm{KBr}, v, \mathrm{~cm}^{-1}\right): 1664(\mathrm{C}=\mathrm{O}), 1592(\mathrm{C}=\mathrm{C}) .{ }^{1} \mathrm{H}$ NMR $(400 \mathrm{MHz}$, $\left.\mathrm{CDCl}_{3}, \delta, \mathrm{ppm}\right): 8.05(\mathrm{~d}, 1 \mathrm{H}, J=12 \mathrm{~Hz}, \beta-\mathrm{CH}), 7.34$ (s, $1 \mathrm{H}, \mathrm{H}-4$ '), 7.29 (s, 1H, H-1'), 7.16 (d, 1H, $J=12 \mathrm{~Hz}, \alpha-\mathrm{CH}), 7.01-6.72(\mathrm{~m}$, 4H, H-2", H-3", H-5", H-6"), 5.97 (s, 2H, OCH $\mathrm{O}$ ), 4.09 (s, 2H, $\left.\mathrm{NH}_{2}\right), 2.18\left(\mathrm{~s}, 3 \mathrm{H}, \mathrm{CH}_{3}\right) .{ }^{13} \mathrm{C} \mathrm{NMR}\left(100 \mathrm{MHz}, \mathrm{CDCl}_{3}, \delta, \mathrm{ppm}\right)$ : $184.4,153.2,148.0,145.6,143.5,137.1,132.6,128.5,128.9$, 123.6, 121.0, 103.9, 101.4, 100.2, 21.5. MS (ESI) m/z: 281.01 $\left(M^{+}\right)$. Anal. Calcd. for $\mathrm{C}_{17} \mathrm{H}_{15} \mathrm{NO}_{3}$ : C, 72.58; H, 5.37; N, 4.98. Found: C, 72.61; H, 5.35; N, 4.99\%.

1-(6-Aminobenzo[d][1,3]dioxol-5-yl)-3-(3,4-dimethylphenyl) prop-2-en-1-one (3h)

Colour: light green solid. Yield: $62.0 \%$. M.p.: 125 $125^{\circ} \mathrm{C}$. IR (KBr, v, $\left.\mathrm{cm}^{-1}\right): 1660(\mathrm{C}=\mathrm{O}), 1596(\mathrm{C}=\mathrm{C}) .{ }^{1} \mathrm{H}$ NMR (400 MHz, $\left.\mathrm{CDCl}_{3}, \delta, \mathrm{ppm}\right): 7.98(\mathrm{~d}, 1 \mathrm{H}, J=13 \mathrm{~Hz}, \beta-\mathrm{CH}), 7.32$ (s, 1H, H-4'), 7.26 (s, 1H, H-1'), 7.13 (d, 1H, J=13 Hz, $\alpha-\mathrm{CH}$ ), 7.12-6.71 (m, 3H, H-2", H-5", H-6"), 6.08 (s, 2H, OCH 2 ), 4.22 $\left(\mathrm{s}, 2 \mathrm{H}, \mathrm{NH}_{2}\right), 2.21\left(\mathrm{~s}, 6 \mathrm{H}, \mathrm{CH}_{3}\right) \cdot{ }^{13} \mathrm{C} \mathrm{NMR}\left(100 \mathrm{MHz}, \mathrm{CDCl}_{3}\right.$, $\delta$, ppm): 186.7, 153.3, 148.5, 144.3, 140.5, 134.4, 130.7, 129.5, $128.7,127.0,124.2,103.0,101.1,100.4,19.6,18.2 . \mathrm{MS}(E S I)$ $\mathrm{m} / \mathrm{z}$ : $295.04\left(M^{+}\right)$. Anal. Calcd. for $\mathrm{C}_{18} \mathrm{H}_{17} \mathrm{NO}_{3}$ : C, 73.20; H, 5.80; N, 4.74. Found: C, 73.21; H, 5.76; N, 4.71\%.

\section{General procedure for the preparation of $N$-phenylamino chalcones (4a-h)}

Dry potassium carbonate dissolved in $5 \mathrm{~mL}$ of amyl alcohol and treated slowly with a mixture of Chalcone (3a-h) ( $5 \mathrm{mmol})$, chlorobenzene $(0.51 \mathrm{~mL}, 5 \mathrm{mmol})$ and copper oxide powder $(0.1 \mathrm{~g})$ were dissolved in $20 \mathrm{~mL}$ of amyl alcohol. The reaction mixture was allowed to reflux for $6 \mathrm{~h}$ at about $100{ }^{\circ} \mathrm{C}$. The amyl alcohol was removed by rotary evaporator then, hot water was added to the reaction mixture and the $\mathrm{pH}$ adjusted to
7 using diluted $\mathrm{HCl}$. The precipitate formed was filtered, washed with cold water and collected. On addition of aqueous solution of sodium hydroxide to the crude product, boiled in the presence of activated charcoal and filtered, the filtrate is acidified with conc. $\mathrm{HCl}$. Upon cooling, the precipitate was obtained, filtered off and recrystallized from ethanol to obtained $N$-phenylamino chalcones (4a-h).

3-Phenyl-1-(6-(phenylamino)benzo[d][1,3]dioxol-5-yl)prop-2en-1-one (4a)

Colour: light brown solid. Yield: $65.0 \%$. M.p.: 132$134^{\circ} \mathrm{C}$. IR $\left(\mathrm{KBr}, v, \mathrm{~cm}^{-1}\right): 1661(\mathrm{C}=\mathrm{O}), 1586(\mathrm{C}=\mathrm{C}) .{ }^{1} \mathrm{H}$ NMR $(400$ $\mathrm{MHz}, \mathrm{CDCl}_{3}, \delta$, ppm): 7.89 (d, $\left.1 \mathrm{H}, J=15 \mathrm{~Hz}, \beta-\mathrm{CH}\right), 7.23-7.13$ (m, 5H, H-2", H-3", H-4", H-5", H-6"), 7.46 (s, 1H, H-4'), 7.33 (s, 1H, H-1'), 7.04 (d, 1H, $J=15 \mathrm{~Hz}, \alpha-\mathrm{CH}), 6.93-6.68$ (m, 5H, $\left.\mathrm{N}-\mathrm{C}_{6} \mathrm{H}_{5}\right), 6.08$ (s, 2H, OCH $\left.\mathrm{H}_{2} \mathrm{O}\right), 4.16$ (s, $\left.1 \mathrm{H}, \mathrm{NH}\right) .{ }^{13} \mathrm{C}$ NMR $(100$ $\left.\mathrm{MHz}, \mathrm{CDCl}_{3}, \delta, \mathrm{ppm}\right): 188.3,154.4,148.5,144.1,142.3,136.8$, $135.8,129.3,128.1,127.5,127.0,126.3,121.5,121.0,120.3$, 103.9, 101.3, 99.1. MS (ESI) m/z: $343.02\left(M^{+}\right)$. Anal. Calcd. for $\mathrm{C}_{22} \mathrm{H}_{17} \mathrm{NO}_{3}$ : C, 76.95; H, 4.99; N, 4.08. Found: C, 76.96; H, 4.97; $\mathrm{N}, 4.05 \%$.

3-(4-Chlorophenyl)-1-(6-(phenylamino)benzo[d][1,3]dioxol-5yl)prop-2-en-1-one (4b)

Colour: light brown solid. Yield: 72.0\%. M.p.: 135$137^{\circ} \mathrm{C}$. IR $\left(\mathrm{KBr}, v, \mathrm{~cm}^{-1}\right): 1668(\mathrm{C}=\mathrm{O}), 1582(\mathrm{C}=\mathrm{C}) .{ }^{1} \mathrm{H}$ NMR (400 MHz, $\left.\mathrm{CDCl}_{3}, \delta, \mathrm{ppm}\right): 7.80$ (d, $\left.1 \mathrm{H}, J=13 \mathrm{~Hz}, \beta-\mathrm{CH}\right), 7.40$ 7.34 (m, 5H, N-C $\left.\mathrm{H}_{5}\right), 7.29$ (s, 1H, H-4'), 7.24 (s, 1H, H-1'), 7.03 (d, $1 \mathrm{H}, J=13 \mathrm{~Hz}, \alpha-\mathrm{CH}$ ), 6.91-6.73 (m, 4H, H-2", H-3”, H-5”, H-6"), 6.12 (s, 2H, OCH $\mathrm{O}), 4.15$ (s, 1H, NH). ${ }^{13} \mathrm{C}$ NMR (100 $\left.\mathrm{MHz}, \mathrm{CDCl}_{3}, \delta, \mathrm{ppm}\right): 188.2,152.2,145.6,145.0,142.7,136.4$, $133.0,132.3,129.1,128.0,127.9,126.3,121.4,121.0,120.7$, 103.2, 101.0, 99.9. MS (ESI) m/z: $377.00\left(M^{+}\right)$. Anal. Calcd. for $\mathrm{C}_{22} \mathrm{H}_{16} \mathrm{ClNO}_{3}$ : C, 69.94; H, 4.27; N, 3.71. Found: C, 69.96; H, $4.36 ; \mathrm{N}, 3.72 \%$.

3-(4-Fluorophenyl)-1-(6-(phenylamino)benzo[d][1,3]dioxol-5-yl) prop-2-en-1-one (4c)

Colour: light brown solid. Yield: $75.0 \%$. M.p.: 138$140^{\circ} \mathrm{C}$. IR (KBr, v, $\left.\mathrm{cm}^{-1}\right)$ : $1660(\mathrm{C}=\mathrm{O}), 1578(\mathrm{C}=\mathrm{C}) .{ }^{1} \mathrm{H}$ NMR (400 MHz, $\left.\mathrm{CDCl}_{3}, \delta, \mathrm{ppm}\right): 8.12$ (d, $\left.1 \mathrm{H}, J=12 \mathrm{~Hz}, \beta-\mathrm{CH}\right), 7.76-$ 7.56 (m, 4H, H-2", H-3", H-5”, H-6”), 7.35 (s, 1H, H-4'), 7.28 (s, 1H, H-1'), $7.11(\mathrm{~d}, 1 \mathrm{H}, J=12 \mathrm{~Hz}, \alpha-\mathrm{CH}), 7.01-6.67$ (m, 5H, $\left.\mathrm{N}-\mathrm{C}_{6} \mathrm{H}_{5}\right), 6.19$ (s, 2H, OCH $\left.\mathrm{O}\right), 4.18(\mathrm{~s}, 1 \mathrm{H}, \mathrm{NH}) .{ }^{13} \mathrm{C}$ NMR $(100$ $\left.\mathrm{MHz}, \mathrm{CDCl}_{3}, \delta, \mathrm{ppm}\right): 185.9,162.8,154.4,148.8,145.6,142.0$, $136.4,130.9,130.2,129.5,126.3,121.9,121.1,120.2,115.2$, 103.2, 101.5, 99.1. MS (ESI) m/z: $361.09\left(M^{+}\right)$. Anal. Calcd. for $\mathrm{C}_{22} \mathrm{H}_{16} \mathrm{FNO}_{3}$ : C, 73.12; H, 4.46; N, 3.88. Found: C, 73.10; H, 4.49; $\mathrm{N}, 3.87 \%$.

3-(4-Nitrophenyl)-1-(6-(phenylamino)benzo[d][1,3]dioxol-5-yl) prop-2-en-1-one (4d)

Colour: light brown solid. Yield: 63.0\%. M.p.: 134$136^{\circ} \mathrm{C}$. IR ( $\left.\mathrm{KBr}, v, \mathrm{~cm}^{-1}\right): 1669(\mathrm{C}=\mathrm{O}), 1585(\mathrm{C}=\mathrm{C}) .{ }^{1} \mathrm{H}$ NMR (400 MHz, $\left.\mathrm{CDCl}_{3}, \delta, \mathrm{ppm}\right): 8.10$ (d, $\left.1 \mathrm{H}, J=14 \mathrm{~Hz}, \beta-\mathrm{CH}\right), 7.62-$ 7.50 (m, 4H, H-2", H-3", H-5", H-6"), 7.20 (s, 1H, H-1'), 6.93 (d, $1 \mathrm{H}, J=14 \mathrm{~Hz}, \alpha-\mathrm{CH}), 6.90-6.69\left(\mathrm{~m}, 5 \mathrm{H}, N-\mathrm{C}_{6} \mathrm{H}_{5}\right), 7.18(\mathrm{~s}, 1 \mathrm{H}$, H-4'), 7.02 (s, 2H, OCH $\mathrm{O}), 4.23$ (s, 1H, NH). ${ }^{13} \mathrm{C}$ NMR (100 
$\left.\mathrm{MHz}, \mathrm{CDCl}_{3}, \delta, \mathrm{ppm}\right): 188.3,154.6,148.2,147.4,145.9,142.0$, $141.6,136.5,129.4,129.0,126.7,123.2,121.9,121.1,120.7$, 103.5, 101.6, 99.3. MS (ESI) m/z: $388.16\left(M^{+}\right)$. Anal. Calcd. for $\mathrm{C}_{22} \mathrm{H}_{16} \mathrm{~N}_{2} \mathrm{O}_{5}$ : C, 68.04; H, 4.15; N, 7.21. Found: C, 68.07; H, 4.13; $\mathrm{N}, 7.20 \%$.

3-(4-methoxyphenyl)-1-(6-(phenylamino)benzo[d][1,3]dioxol-5yl)prop-2-en-1-one (4e)

Colour: light brown solid. Yield: 78.0\%. M.p.: 141$143^{\circ} \mathrm{C}$. IR $\left(\mathrm{KBr}, v, \mathrm{~cm}^{-1}\right): 1665(\mathrm{C}=\mathrm{O}), 1588(\mathrm{C}=\mathrm{C}) .{ }^{1} \mathrm{H}$ NMR $(400$ $\left.\mathrm{MHz}, \mathrm{CDCl}_{3}, \delta, \mathrm{ppm}\right): 8.02(\mathrm{~d}, 1 \mathrm{H}, J=12 \mathrm{~Hz}, \beta-\mathrm{CH}), 7.54-7.36$ (m, 4H, H-2", H-3", H-5”, H-6”), 7.34 (s, 1H, H-4'), 7.26 (s, 1H, H-1'), 7.14 (d, 1H, $J=12 \mathrm{~Hz}, \alpha-\mathrm{CH}), 7.03-6.75$ (m, 5H, $N-\mathrm{C}_{6} \mathrm{H}_{5}$ ), $6.12\left(\mathrm{~s}, 2 \mathrm{H}, \mathrm{OCH}_{2} \mathrm{O}\right), 4.19$ (s, $\left.1 \mathrm{H}, \mathrm{NH}\right), 3.89$ (s, 3H, $\left.\mathrm{OCH}_{3}\right) .{ }^{13} \mathrm{C}$ NMR (100 MHz, $\left.\mathrm{CDCl}_{3}, \delta, \mathrm{ppm}\right): 188.5,159.2,154.4,148.7$, $145.4,142.6,136.0,130.5,129.2,127.8,126.1,121.6,121.0$, 120.2, 114.5, 103.5, 101.6, 99.6, 55.5. MS (ESI) m/z: $373.15\left(M^{+}\right)$. Anal. Calcd. for $\mathrm{C}_{23} \mathrm{H}_{19} \mathrm{NO}_{4}$ : C, 73.98; H, 5.13; N, 3.75. Found: C, 73.95; H, 5.15; N, 3.71\%.

1-(6-(Phenylamino)benzo[d][1,3]dioxol-5-yl)-3-(3,4,5trimethoxyphenyl)prop-2-en-1-one (4f)

Colour: light brown solid. Yield: 78.0\%. M.p.: 143$145^{\circ} \mathrm{C}$. IR $\left(\mathrm{KBr}, v, \mathrm{~cm}^{-1}\right): 1661(\mathrm{C}=\mathrm{O}), 1582(\mathrm{C}=\mathrm{C}) .{ }^{1} \mathrm{H}$ NMR (400 MHz, $\left.\mathrm{CDCl}_{3}, \delta, \mathrm{ppm}\right): 7.89$ (d, $\left.1 \mathrm{H}, J=13 \mathrm{~Hz}, \beta-\mathrm{CH}\right), 7.41$ (s, 1H, H-4'), 7.34-7.12 (m, 5H, N-C $\mathrm{C}_{5}$ ), 7.03 (s, 1H, H-1'), 6.85 (d, 1H, J = $13 \mathrm{~Hz}, \alpha-\mathrm{CH}), 6.72$ (s, 2H, H-2", H-6"), 6.09 (s, 2H, $\left.\mathrm{OCH}_{2} \mathrm{O}\right), 4.17(\mathrm{~s}, 1 \mathrm{H}, \mathrm{NH}), 3.86\left(\mathrm{~s}, 9 \mathrm{H}, \mathrm{OCH}_{3}\right) \cdot{ }^{13} \mathrm{C} \mathrm{NMR}(100$ $\left.\mathrm{MHz}, \mathrm{CDCl}_{3}, \delta, \mathrm{ppm}\right): 186.3,154.4,152.0,148.0,142.7,145.5$, $138.2,136.9,129.5,126.9,126.2,121.5,121.0,120.3,103.5$, 103.0, 101.7, 99.5, 60.4, 56.4. MS (ESI) m/z: $433.13\left(M^{+}\right)$. Anal. Calcd. for $\mathrm{C}_{25} \mathrm{H}_{23} \mathrm{NO}_{6}$ : C, 69.27; H, 5.35; N, 3.23. Found: C, 69.29; H, 5.32; N, 3.20\%.

1-(6-(Phenylamino)benzo[d][1,3]dioxol-5-yl)-3-(p-tolyl)prop-2en-1-one (4g)

Colour: light brown solid. Yield: 70.0\%. M.p.: 131$133^{\circ} \mathrm{C}$. IR (KBr, v, $\left.\mathrm{cm}^{-1}\right)$ : $1669(\mathrm{C}=\mathrm{O}), 1591(\mathrm{C}=\mathrm{C}) .{ }^{1} \mathrm{H}$ NMR (400 MHz, $\left.\mathrm{CDCl}_{3}, \delta, \mathrm{ppm}\right): 8.05(\mathrm{~d}, 1 \mathrm{H}, J=15 \mathrm{~Hz}, \beta-\mathrm{CH}), 7.30-$ 7.19 (m, 5H, N-C $\mathrm{H}_{5}$ ), 7.28 (s, 1H, H-4'), 7.13 (s, 1H, H-1'), 7.04 (d, $1 \mathrm{H}, J=15 \mathrm{~Hz}, \alpha-\mathrm{CH}), 6.84-6.62$ (m, 4H, H-2", H-3", H-5", H-6"), 6.02 (s, 2H, $\left.\mathrm{OCH}_{2} \mathrm{O}\right), 4.05$ (s, 1H, NH), 2.36 (s, 3H, $\mathrm{CH}_{3}$ ). ${ }^{13} \mathrm{C}$ NMR $\left(100 \mathrm{MHz}, \mathrm{CDCl}_{3}, \delta, \mathrm{ppm}\right): 188.7,154.1,148.5,145.0$, $142.6,137.5,136.8,132.5,129.2,128.7,128.3,126.1,121.5$, 121.0, 120.4, 103.3, 101.8, 99.9, 21.6. MS (ESI) m/z: $357.17\left(M^{+}\right)$. Anal. Calcd. for $\mathrm{C}_{23} \mathrm{H}_{19} \mathrm{NO}_{3}$ : C, 77.29; H, 5.36; N, 3.92. Found: C, 77.27; H, 5.38; N, 3.93\%.

3-(3,4-Dimethylphenyl)-1-(6-(phenylamino)benzo[d][1,3]dioxol5-yl)prop-2-en-1-one (4h)

Colour: light brown solid. Yield: $65.0 \%$. M.p.: 148$150^{\circ} \mathrm{C}$. IR $\left(\mathrm{KBr}, v, \mathrm{~cm}^{-1}\right): 1665(\mathrm{C}=\mathrm{O}), 1587(\mathrm{C}=\mathrm{C}) .{ }^{1} \mathrm{H}$ NMR $(400$ $\left.\mathrm{MHz}, \mathrm{CDCl}_{3}, \delta, \mathrm{ppm}\right): 7.94$ (d, $\left.1 \mathrm{H}, J=13 \mathrm{~Hz}, \beta-\mathrm{CH}\right), 7.35-7.22$ $\left(\mathrm{m}, 5 \mathrm{H}, \mathrm{N}-\mathrm{C}_{6} \mathrm{H}_{5}\right), 7.46$ (s, 1H, H-4'), 7.12 (s, 1H, H-1'), 6.93 (d, $1 \mathrm{H}, J=13 \mathrm{~Hz}, \alpha-\mathrm{CH}), 6.83-6.79$ (m, 3H, H-2", H-5", H-6"), 6.12 $\left(\mathrm{s}, 2 \mathrm{H}, \mathrm{OCH}_{2} \mathrm{O}\right), 4.16(\mathrm{~s}, 1 \mathrm{H}, \mathrm{NH}), 2.28\left(\mathrm{~s}, 6 \mathrm{H}, \mathrm{CH}_{3}\right) .{ }^{13} \mathrm{C} \mathrm{NMR}$ (100 MHz, $\left.\mathrm{CDCl}_{3}, \delta, \mathrm{ppm}\right): 187.4,154.4,148.8,142.2,140.0$, $136.6,134.9,130.3,129.8,129.0,128.5,127.0,126.6,121.6$, 120.2, 103.0, 101.5, 99.1, 19.8, 18.6. MS $(E S I) \mathrm{m} / \mathrm{z}: 371.10\left(M^{+}\right)$.
Anal. Calcd. for $\mathrm{C}_{24} \mathrm{H}_{21} \mathrm{NO}_{3}$ : C, 77.61; H, 5.70; N, 3.77. Found: C, $77.63 ; \mathrm{H}, 5.68 ; \mathrm{N}, 3.74 \%$.

General procedure for the preparation of $N$-phenyl tetralones (5a-h)

To the well-stirred mixture of compounds (4a-h) (1 mmol) and orthophosphoric acid $(10 \mathrm{~mL})$ in glacial acetic acid $(20 \mathrm{~mL})$, then the reaction mixture was reflux for $2 \mathrm{~h}$. After the completion of the reaction, the reaction mass was quenched in an ice-cold water and extracted in chloroform. The chloroform layer was washed twice with a saturated solution of sodium bicarbonate and twice with distilled water. Finally, the ether layer was dried over anhydrous $\mathrm{Na}_{2} \mathrm{SO}_{4}$. The solvent was evaporated under reduced pressure and the obtained product was recrystallized by using ethanol.

5,6-Diphenyl-6,7-dihydro-[1,3]dioxolo[4,5-g]quinolin-8(5H)one (5a)

Colour: light brown solid. Yield: $54.0 \%$. M.p.: $147-$ $149^{\circ} \mathrm{C}$. IR $\left(\mathrm{KBr}, v, \mathrm{~cm}^{-1}\right): 1681(\mathrm{C}=\mathrm{O}) .{ }^{1} \mathrm{H}$ NMR $(400 \mathrm{MHz}$, $\left.\mathrm{CDCl}_{3}, \delta, \mathrm{ppm}\right): 7.50-7.42\left(\mathrm{~m}, 5 \mathrm{H}, \mathrm{N}-\mathrm{C}_{6} \mathrm{H}_{5}\right), 7.24(\mathrm{~s}, 1 \mathrm{H}, \mathrm{H}-8)$, 6.92 (s, 1H, H-5), 6.74-6.56 (m, 5H, H-2', H-3', H-4', H-5', H-6'), $6.17\left(\mathrm{~s}, 2 \mathrm{H}, \mathrm{OCH}_{2} \mathrm{O}\right), 4.38(\mathrm{t}, 1 \mathrm{H}, J=3.2 \mathrm{~Hz}, \mathrm{CH}), 2.73(\mathrm{dd}, 2 \mathrm{H}$, $\left.J=2.1 \mathrm{~Hz}, \mathrm{CH}_{2}\right) .{ }^{13} \mathrm{C} \mathrm{NMR}\left(100 \mathrm{MHz}, \mathrm{CDCl}_{3}, \delta, \mathrm{ppm}\right): 190.2$, $152.1,149.4,142.5,137.4,136.7,129.3,127.3,126.5,126.1$, 121.3, 119.8, 114.0, 103.5, 104.4, 101.8, 65.6, 44.7. MS (ESI) m/z: $343.10\left(M^{+}\right)$. Anal. Calcd. for $\mathrm{C}_{22} \mathrm{H}_{17} \mathrm{NO}_{3}: \mathrm{C}, 76.95 ; \mathrm{H}, 4.99 ; \mathrm{N}$, 4.08. Found: C, 76.91; H, 5.02; N, 4.05\%.

6-(4-Chlorophenyl)-5-phenyl-6,7-dihydro-[1,3]dioxolo[4,5-g] quinolin-8(5H)-one (5b)

Colour: light brown solid. Yield: 57.0\%. M.p.: 154$156^{\circ} \mathrm{C}$. IR (KBr, v, cm $\left.{ }^{-1}\right): 1664(\mathrm{C}=\mathrm{O}) .{ }^{1} \mathrm{H}$ NMR $(400 \mathrm{MHz}$, $\left.\mathrm{CDCl}_{3}, \delta, \mathrm{ppm}\right): 7.40-7.32\left(\mathrm{~m}, 5 \mathrm{H}, \mathrm{N}-\mathrm{C}_{6} \mathrm{H}_{5}\right), 7.28(\mathrm{~s}, 1 \mathrm{H}, \mathrm{H}-8)$, 6.88-6.70 (m, 4H, H-2', H-3', H-5', H-6'), 6.16 (s, 1H, H-5), 6.12 $\left(\mathrm{s}, 2 \mathrm{H}, \mathrm{OCH}_{2} \mathrm{O}\right), 4.72(\mathrm{t}, 1 \mathrm{H}, J=4.1 \mathrm{~Hz}, \mathrm{CH}), 2.41(\mathrm{dd}, 2 \mathrm{H}, J=$ $\left.2.8 \mathrm{~Hz}, \mathrm{CH}_{2}\right) .{ }^{13} \mathrm{C} \mathrm{NMR}\left(100 \mathrm{MHz}, \mathrm{CDCl}_{3}, \delta, \mathrm{ppm}\right): 191.3,152.3$, $148.4,141.2,138.8,136.7,132.5,129.3,128.1,127.6,121.1$, 119.4, 114.6, 108.0, 103.7, 101.5, 65.7, 44.1. MS (ESI) m/z: $377.09\left(M^{+}\right)$. Anal. Calcd. for $\mathrm{C}_{22} \mathrm{H}_{16} \mathrm{ClNO}_{3}: \mathrm{C}, 69.94 ; \mathrm{H}, 4.27 ; \mathrm{N}$, 3.71. Found: C, 69.91; H, 4.29; N, 3.73\%.

6-(4-Fluorophenyl)-5-phenyl-6,7-dihydro-[1,3]dioxolo[4,5-g] quinolin-8(5H)-one (5c)

Colour: light brown solid. Yield: 51.0\%. M.p.: 157$159^{\circ} \mathrm{C}$. IR $\left(\mathrm{KBr}, v, \mathrm{~cm}^{-1}\right): 1653(\mathrm{C}=\mathrm{O}) .{ }^{1} \mathrm{H}$ NMR $(400 \mathrm{MHz}$, $\mathrm{CDCl}_{3}, \delta, \mathrm{ppm}$ ): 7.68-7.54 (m, 4H, H-2', H-3', H-5', H-6'), 7.30 (s, 1H, H-8), $7.13(\mathrm{~s}, 1 \mathrm{H}, \mathrm{H}-5), 6.93-6.67\left(\mathrm{~m}, 5 \mathrm{H}, \mathrm{N}-\mathrm{C}_{6} \mathrm{H}_{5}\right), 6.16$ $\left(\mathrm{s}, 2 \mathrm{H}, \mathrm{OCH}_{2} \mathrm{O}\right), 4.65$ (t, $\left.1 \mathrm{H}, J=3.8 \mathrm{~Hz}, \mathrm{CH}\right), 2.38$ (dd, $2 \mathrm{H}, J=$ $\left.3.0 \mathrm{~Hz}, \mathrm{CH}_{2}\right) .{ }^{13} \mathrm{C} \mathrm{NMR}\left(100 \mathrm{MHz} \mathrm{CDCl}_{3}, \delta\right.$, ppm): 192.4, 160.4, $152.1,149.5,139.7,137.2,135.2,129.3,128.8,121.1,119.6$, $115.0,114.8,108.2,103.4,101.6,65.6,44.9 . M S(E S I) \mathrm{m} / \mathrm{z}$ : $361.14\left(M^{+}\right)$. Anal. Calcd. for $\mathrm{C}_{22} \mathrm{H}_{16} \mathrm{FNO}_{3}$ : C, 73.12; $\mathrm{H}, 4.46 ; \mathrm{N}$, 3.88. Found: C, 73.14 ; H, 4.45; N, 3.85\%.

6-(4-Nitrophenyl)-5-phenyl-6,7-dihydro-[1,3]dioxolo[4,5-g] quinolin-8(5H)-one (5d)

Colour: light brown solid. Yield: 47.0\%. M.p.: 142$144^{\circ} \mathrm{C}$. IR $\left(\mathrm{KBr}, v, \mathrm{~cm}^{-1}\right)$ : $1658(\mathrm{C}=\mathrm{O}) .{ }^{1} \mathrm{H}$ NMR $(400 \mathrm{MHz}$, 
$\left.\mathrm{CDCl}_{3}, \delta, \mathrm{ppm}\right)$ : 7.88-7.72 (m, 4H, H-2', H-3', H-5', H-6'), 7.48$7.36\left(\mathrm{~m}, 5 \mathrm{H}, \mathrm{N}-\mathrm{C}_{6} \mathrm{H}_{5}\right), 7.30(\mathrm{~s}, 1 \mathrm{H}, \mathrm{H}-8), 7.15(\mathrm{~s}, 1 \mathrm{H}, \mathrm{H}-5), 6.11$ $\left(\mathrm{s}, 2 \mathrm{H}, \mathrm{OCH}_{2} \mathrm{O}\right), 4.60(\mathrm{t}, 1 \mathrm{H}, J=3.1 \mathrm{~Hz}, \mathrm{CH}), 2.31(\mathrm{dd}, 2 \mathrm{H}, J=$ $\left.4.4 \mathrm{~Hz}, \mathrm{CH}_{2}\right) .{ }^{13} \mathrm{C} \mathrm{NMR}\left(100 \mathrm{MHz}, \mathrm{CDCl}_{3}, \delta\right.$, ppm): 193.5, 152.1, $149.4,149.0,145.3,137.7,136.6,129.1,123.5,123.1,118.3$, $115.6,114.8,108.2,103.6,101.3,65.7,43.8 . \mathrm{MS}(E S I) \mathrm{m} / \mathrm{z}$ : $388.15\left(M^{+}\right)$. Anal. Calcd. for $\mathrm{C}_{22} \mathrm{H}_{16} \mathrm{~N}_{2} \mathrm{O}_{5}: \mathrm{C}, 68.04 ; \mathrm{H}, 4.15 ; \mathrm{N}$, 7.21. Found: C, 68.00; H, 4.17; N, 7.20\%.

6-(4-Methoxyphenyl)-5-phenyl-6,7-dihydro-[1,3]dioxolo[4,5-g] quinolin-8(5H)-one (5e)

Colour: light brown solid. Yield: $68.0 \%$. M.p.: 158$160^{\circ} \mathrm{C}$. IR (KBr, v, $\left.\mathrm{cm}^{-1}\right): 1669(\mathrm{C}=\mathrm{O}) .{ }^{1} \mathrm{H}$ NMR $(400 \mathrm{MHz}$, $\mathrm{CDCl}_{3}, \delta, \mathrm{ppm}$ ): 7.67-7.47 (m, 4H, H-2', H-3', H-5', H-6'), 7.37$7.25\left(\mathrm{~m}, 5 \mathrm{H}, \mathrm{N}-\mathrm{C}_{6} \mathrm{H}_{5}\right), 7.21(\mathrm{~s}, 1 \mathrm{H}, \mathrm{H}-8), 6.93$ (s, 1H, H-5), 6.19 $\left(\mathrm{s}, 2 \mathrm{H}, \mathrm{OCH}_{2} \mathrm{O}\right), 4.45$ (t, $\left.1 \mathrm{H}, J=3.7 \mathrm{~Hz}, \mathrm{CH}\right), 3.87\left(\mathrm{~s}, 3 \mathrm{H}, \mathrm{OCH}_{3}\right)$, $2.25\left(\mathrm{dd}, 2 \mathrm{H}, J=2.3 \mathrm{~Hz}, \mathrm{CH}_{2}\right) .{ }^{13} \mathrm{C} \mathrm{NMR}\left(100 \mathrm{MHz}, \mathrm{CDCl}_{3}, \delta\right.$, ppm): 193.7, 156.1, 152.0, 145.1, 137.9, 137.1, 136.3, 128.3, $126.1,121.5,117.1,114.9,114.3,106.8,103.5,101.7,65.5,55.2$, 44.7. MS (ESI) m/z: $373.15\left(M^{+}\right)$. Anal. Calcd. for $\mathrm{C}_{23} \mathrm{H}_{19} \mathrm{NO}_{4}$ : C, 73.98; H, 5.13; N, 3.75. Found: C, 74.01; H, 5.11; N, 3.72\%.

5-Phenyl-6-(3,4,5-trimethoxyphenyl)-6,7-dihydro-[1,3]

dioxolo[4,5-g]quinolin-8(5H)-one (5f)

Colour: light brown solid. Yield: 75.0\%. M.p.: $167-$ $169^{\circ} \mathrm{C} . \mathrm{IR}\left(\mathrm{KBr}, v, \mathrm{~cm}^{-1}\right): 1654(\mathrm{C}=\mathrm{O}) .{ }^{1} \mathrm{H} \mathrm{NMR}\left(400 \mathrm{MHz}, \mathrm{CDCl}_{3}\right.$, $\delta$, ppm): 7.36 (s, 2H, H-2', H-6'), 7.25-7.13 (m, 5H, N-C $\mathrm{H}_{5}$ ), 7.29 (s, 1H, H-8), 6.80 (s, 1H, H-5), 6.16 (s, 2H, $\left.\mathrm{OCH}_{2} \mathrm{O}\right), 4.49$ (t, 1H, $J=3.2 \mathrm{~Hz}, \mathrm{CH}), 3.89\left(\mathrm{~s}, 9 \mathrm{H}, \mathrm{OCH}_{3}\right), 2.37$ (dd, $2 \mathrm{H}, J=3.5 \mathrm{~Hz}$, $\left.\mathrm{CH}_{2}\right) \cdot{ }^{13} \mathrm{C}$ NMR $\left(100 \mathrm{MHz}, \mathrm{CDCl}_{3}, \delta, \mathrm{ppm}\right): 191.8,150.5,150.0$, $147.6,138.6,137.2,137.5,136.6,127.6,121.7,119.4,114.6$, 108.1, 103.5, 102.7, 101.3, 65.5, 60.0, 56.3, 44.2. MS (ESI) m/z: $433.12\left(M^{+}\right)$. Anal. Calcd. for $\mathrm{C}_{25} \mathrm{H}_{23} \mathrm{NO}_{6}: \mathrm{C}, 69.27 ; \mathrm{H}, 5.35 ; \mathrm{N}$, 3.23. Found: C, 69.29; H, 5.31; N, 3.24\%.

5-Phenyl-6-(p-tolyl)-6,7-dihydro-[1,3]dioxolo[4,5-g]quinolin$8(5 H)$-one $(5 g)$

Colour: light brown solid. Yield: 71.0\%. M.p.: 163$165^{\circ} \mathrm{C}$. IR (KBr, v, $\left.\mathrm{cm}^{-1}\right): 1662(\mathrm{C}=\mathrm{O}) .{ }^{1} \mathrm{H}$ NMR $(400 \mathrm{MHz}$, $\mathrm{CDCl}_{3}, \delta$, ppm): 7.26-7.08 (m, 4H, H-2', H-3', H-5', H-6'), 6.84$6.67\left(\mathrm{~m}, 5 \mathrm{H}, \mathrm{N}-\mathrm{C}_{6} \mathrm{H}_{5}\right), 7.32$ (s, 1H, H-8), 6.65 (s, 1H, H-5), 6.19 $\left(\mathrm{s}, 2 \mathrm{H}, \mathrm{OCH}_{2} \mathrm{O}\right), 4.41(\mathrm{t}, 1 \mathrm{H}, J=4.2 \mathrm{~Hz}, \mathrm{CH}), 2.33$ (dd, 2H, $J=$ $\left.3.0 \mathrm{~Hz}, \mathrm{CH}_{2}\right), 2.23\left(\mathrm{~s}, 3 \mathrm{H}, \mathrm{CH}_{3}\right) .{ }^{13} \mathrm{C} \mathrm{NMR} \mathrm{(100} \mathrm{MHz,} \mathrm{CDCl}_{3}$, $\delta, \mathrm{ppm}): 193.8,152.6,149.5,140.5,138.3,136.4,136.0,128.1$, 128.3, 125.8, 121.6, 118.5, 114.7, 108.3, 103.6, 101.3, 65.0, 44.7, 21.8. MS (ESI) m/z: $357.11\left(M^{+}\right)$. Anal. Calcd. for $\mathrm{C}_{23} \mathrm{H}_{19} \mathrm{NO}_{3}: \mathrm{C}$, 77.29; H, 5.36; N, 3.92. Found: C, 77.32; H, 5.32; N, 3.95\%.

6-(3,4-Dimethylphenyl)-5-phenyl-6,7-dihydro-[1,3]

dioxolo[4,5-g]quinolin-8(5H)-one (5h)

Colour: light brown solid. Yield: $66.0 \%$. M.p.: 148$150^{\circ} \mathrm{C}$. IR (KBr, v, $\left.\mathrm{cm}^{-1}\right): 1659(\mathrm{C}=\mathrm{O}) .{ }^{1} \mathrm{H}$ NMR $(400 \mathrm{MHz}$, $\left.\mathrm{CDCl}_{3}, \delta, \mathrm{ppm}\right)$ : 7.33-7.12 (m, 3H, H-2', H-5', H-6'), 6.83-6.69 $\left(\mathrm{m}, 5 \mathrm{H}, \mathrm{N}-\mathrm{C}_{6} \mathrm{H}_{5}\right), 7.42(\mathrm{~s}, 1 \mathrm{H}, \mathrm{H}-8), 7.08$ (s, 1H, H-5), 6.13 (s, $\left.2 \mathrm{H}, \mathrm{OCH}_{2} \mathrm{O}\right), 4.43(\mathrm{t}, 1 \mathrm{H}, J=3.9 \mathrm{~Hz}, \mathrm{CH}), 2.28(\mathrm{dd}, 2 \mathrm{H}, J=$ $\left.4.0 \mathrm{~Hz}, \mathrm{CH}_{2}\right), 2.26\left(\mathrm{~s}, 6 \mathrm{H}, \mathrm{CH}_{3}\right) .{ }^{13} \mathrm{C} \mathrm{NMR}\left(100 \mathrm{MHz}, \mathrm{CDCl}_{3}\right.$, $\delta, \mathrm{ppm}): 192.9,153.2,144.9,138.0,136.9,129.4,129.1,128.5$, 127.2 , 126.5, 121.3, 114.5, 107.9, 103.4, 102.5, 101.0, 65.4, 44.1.
MS (ESI) m/z: $371.18\left(M^{+}\right)$. Anal. Calcd. for $\mathrm{C}_{24} \mathrm{H}_{21} \mathrm{NO}_{3}$ : C, 77.61; H, 5.70; N, 3.77. Found: C, 77.62; H, 5.66; N, 3.73\%.

\section{Antimitotic studies}

The evaluation of antimitotic activity of synthesized compounds was done by onion root tip method. Materials and chemicals required for this examination are compound microscope, glass slides, cover slips, samples $(0.1 \mathrm{mg} / \mathrm{mL})$, acetoorcein solution, Carney's solution II, 3\% ethanol, and hydrochloric acid $(0.1 \mathrm{~N})$. Onion base was immersed to a degree of about a large portion of a centimeter in a sample tube and control solution tube in a glass $(7 \times 3)$, after removing the old roots, inundation proceeds for $24 \mathrm{~h}$ for germination. After this, the sprouted root tips were evacuated and settled in Carney's answer II (alcohol and acetic acid in 3:1 ratio respectively) for $24 \mathrm{~h}$. After $24 \mathrm{~h}$ Carney's answer II was emptied precisely and the root tips were washed with preserving solvent ( $70 \%$ ethanol). The fixed root tips were continued on in $70 \%$ ethanol in the refrigerator. At that point, the root tips were taken in a watch glass and recolored with a drop of acetoorcein stain and a drop of $1 \mathrm{~N} \mathrm{HCl}$ (7:1) was additionally included. The glass slides were warmed and kept for $1 \mathrm{~h}$. The roots were gone up against a spotless glass slide and squashed utilizing 45\% acetic acid (Levan and Hereditas, 1938). A microscope cover glass was set on the material and after that weight was connected to a cover glass to ensure uniform spreading. The cover glass was fixed with molten paraffin wax and the slide was seen under a microscope and photographed. Mitotic Index (M. I.) was calculated (Fissceja and Hereditas, 1985). The mitotic index was determined by examination of a minimum of zone cells. Three replicates were made for each calculation.

$$
\text { M. I. }=\frac{\text { Total number of dividing cells }}{\text { Total number of cells examined }} \times 100 .
$$

The percentage of the number of dividing cells compared to the control and the percent inhibition of mitosis by an antimitotic agent at a concentration $(0.1 \mathrm{mg} / \mathrm{mL})$ against a control was calculated (Hakala et al., 1976).

\section{RESULTS AND DISCUSSION}

\section{Chemistry}

The synthesis of $N$-phenyl tetralones (5a-h) has been carried out by chalcone route (scheme 1). The benzylideneacetophenones (chalcones) (3a-h) were prepared in high yields by Claisen-Schmidt condensation reaction of 5-Acetyl-6-amino-1,3-benzodioxole (1) with aldehydes (2a-h) in the presence of sodium hydroxide in the water-methanol mixture (Umesha and Basavaraju, 2014). The reactions of chlorobenzene and chalcones (3a-h) were brought by refluxing in the presence of copper oxide and potassium carbonate in amyl alcohol medium afforded $N$-phenylamino chalcones (4a-h) in good yields (Khoza et al., 2012). This reaction is a copper-catalyzed aromatic nucleophilic substitution with an aryl halide. $N$-phenylamino chalcones $(\mathbf{4 a}-\mathbf{h})$ were subjected to acid-mediated cyclization with the orthophosphoric-acetic acid mixture to afford $N$-phenyl tetralones (5a-h) (Scheme 1) (Salimon et al., 2010).

Reagents and condition: (a) substituted benzaldehydes (2a-h), 30\% methanolic $\mathrm{NaOH}$, water, r, t; (b) $\mathrm{CuO} / \mathrm{K}_{2} \mathrm{CO}_{3}$, chlorobenzene, amyl alcohol; (c) $\mathrm{H}_{3} \mathrm{PO}_{4}-\mathrm{AcOH}, 110^{\circ} \mathrm{C}$. 


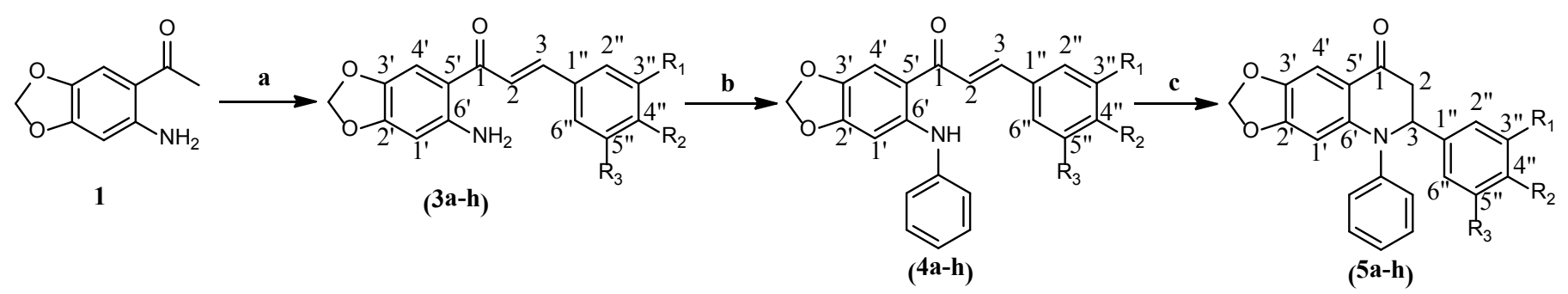

Scheme 1: Synthesis pathway for synthesis of $N$-phenyl tetralones (5a-h).

\section{Antimitotic activity}

Allium Cepa has been utilized to assess the antimitotic activity of $N$-phenyl tetralones (5a-h) by onion root tip method. Onion root tip cells in the solution of synthesized compounds displayed changes in cell morphology, for example, slight prolongation fit as a fiddle with huge numbers of them stay in the soonest phases of mitosis called prophase stage. Onion establishes in integrated mixes of $0.1 \mathrm{mg} / \mathrm{mL}$ at $24 \mathrm{~h}$ displayed changes fit as a fiddle of the cells with lengthened appearance. Cytotoxic nature of $N$-phenyl tetralones indicated less number of partitioning cells.

The result of the antimitotic activity of the different compounds of $N$-phenyl tetralones appeared to be related to the nature of nitrogen present in the ring $\mathrm{C}, 1,3$-benzodioxole unit and substituents on the phenyl ring. The aftereffects of antimitotic activity uncovered that most of the synthesized compounds demonstrated differing \% inhibition compared to control. Compounds 5e and $\mathbf{5 f}$ having electron donating methoxy group(s) observed to be basic to show intense antimitotic activity at para and 3,4,5-positions separately on phenyl ring. Compound 5g containing methyl group on para position of phenyl ring and $\mathbf{5 h}$ having methyl gather at 3,4-positions of phenyl ring showed moderate antimitotic activity took after by $\mathbf{5 a}$ having no group on phenyl ring. Whatever is left of the compounds $\mathbf{5 b}, \mathbf{5 c}$ and $\mathbf{5 d}$ indicated less antimitotic activity as a result of the nearness of electron withdrawing substituent especially chloro, fluoro, and nitro assemble at para position of phenyl ring. The $\%$ Inhibition is exhibited in table 1.

Table 1: \% Inhibition of the synthesized compounds (5a-h) compared to control by onion root method.

\begin{tabular}{|c|c|c|c|c|c|c|}
\hline Compounds & & Entry R & & $\%$ Dividing cells & \% Dividing cells compared to control & $\%$ Inhibition \\
\hline Control & $\mathrm{R}_{1}$ & $\mathrm{R}_{2}$ & $\mathrm{R}_{3}$ & 39.28 & 100 & 100 \\
\hline $5 a$ & $\mathrm{H}$ & $\mathrm{H}$ & $\mathrm{H}$ & 15.92 & 40.53 & 59.47 \\
\hline $5 \mathbf{b}$ & $\mathrm{H}$ & $\mathrm{Cl}$ & $\mathrm{H}$ & 19.74 & 50.25 & 49.75 \\
\hline $5 c$ & $\mathrm{H}$ & $\mathrm{F}$ & $\mathrm{H}$ & 23.18 & 59.01 & 40.99 \\
\hline $5 d$ & $\mathrm{H}$ & $\mathrm{NO}_{2}$ & $\mathrm{H}$ & 21.19 & 53.94 & 46.06 \\
\hline $5 e$ & $\mathrm{H}$ & $\mathrm{OCH}_{3}$ & $\mathrm{H}$ & 10.01 & 25.43 & 74.57 \\
\hline $5 f$ & $\mathrm{OCH}_{3}$ & $\mathrm{OCH}_{3}$ & $\mathrm{OCH}_{3}$ & 8.87 & 22.58 & 77.42 \\
\hline $5 \mathrm{~g}$ & $\mathrm{H}$ & $\mathrm{CH}_{3}$ & $\mathrm{H}$ & 15.11 & 38.46 & 61.54 \\
\hline $5 \mathrm{~h}$ & $\mathrm{H}$ & $\mathrm{CH}_{3}$ & $\mathrm{CH}_{3}$ & 12.37 & 31.49 & 68.51 \\
\hline
\end{tabular}

\section{CONCLUSION}

We have reported a convenient protocol for the synthesis

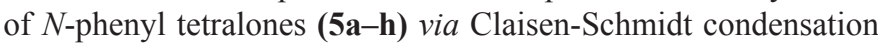
reaction of 5-Acetyl-6-amino-1,3-benzodioxole with different aldehydes in the presence of sodium hydroxide to obtain chalcones followed by copper oxide and potassium carbonate catalyzed aromatic nucleophilic substitution reaction and acidmediated cyclization. The synthesized compounds were screened for their antimitotic capacity. It is noteworthy that compounds $\mathbf{5 e}$ and $\mathbf{5 f}$ exhibited excellent antimitotic capacity compared to other synthesized compounds because they contain an electron donating methoxy group. Our results of these compounds are helpful for further studies in design and discovery of more potent antimitotic agents.

\section{FINANCIAL SUPPORT AND SPONSORSHIP}

The authors are grateful to the University Grants
Commission, Government of India, for granting UGC-Post Doctoral Fellowship SC/ST (PDFSS).

\section{CONFLICT OF INTERESTS}

There are no conflicts of interest.

\section{REFERENCES}

Damayanthi Y, Lown JW. Podophyllotoxins: current status and recent developments. Curr Med Chem. 1998; 5:205-252.

Shi F, Zeng X-N, Zhang G, Ma N, Jiang B, Tu S. Facile synthesis of new 4-aza-podophyllotoxin analogs via microwave-assisted multicomponent reactions and evaluation of their cytotoxic activity. Bioorganic Med Chem Lett. 2011; 21:7119-7123.

Fissceja G. The Allium test as a standard in environmental monitoring. Hereditas. 1985; 102:99-112.

Gordaliza M, Castro MA, Miguel del Corral JM, San Feliciano A. Antitumor properties of podophyllotoxin and related compounds. Curr Pharm Design. 2000; 6:1811-1839.

Hakala TR, Lange PA, Fraley EF. 1976. Method in vitro in all 
mediated and tumor immunity. In: Bloom BR, David JR, ed. Academic Press, New York, 451.

Hande KR. Etoposide: four decades of development of a topoisomerase II inhibitor. Eur J Cancer. 1998; 34:1514-1521.

Jackson DE, Dewick PM. Tumor-inhibitory aryltetralin lignans from podophyllum pleianthum. Phytochemistry. 1985; 24:2407-2409.

Salimon J, Salih N, Yousif E, Hameed A, Kreem A. Synthesis and pharmacological evaluation of $9(10 \mathrm{H})$-acridone bearing 1,3,4-oxadiazole derivatives as antimicrobial agents. Arab J Chem. 2010; 3:205-210.

Lee CTL, Lin VCK, Zhang SX, Zhu XK, Van Vliet D, Hu H, Beers SA, Wang ZQ, Cosentino LM, Morris-Natschke SL, Lee KH. Antiaids agents-29-anti-hiv activity of modified podophyllotoxin derivatives. Bioorganic Med Chem Lett. 1997; 7:2897-2902.

Levan A. The effect of colchicine on root mitoses in allium. Hereditas. 1938; 24:471-486.

Rivera G, Avery T, Pratt C. 4'-Demethylepipodophyllotoxin 9-(4,6-O-2-Thenylidene-Beta-D-Glucopyranoside) (NSC-122819; VM26) and 4'-Demethylepipodophyllotoxin 9-(4.6-0-Ethylidene-Beta-D-
Glucopyranoside) (NSC-141540; VP-16-213) in Childhood Cancer: Preliminary Observations. Cancer Chemother Rep. 1975; 59:743-749.

Khoza TA, Maluleka MM, Mama N, Mphahlele MJ. Synthesis and photophysical properties of 2-aryl-6,8-bis(arylethenyl)-4methoxyquinolines. Molecules. 2012; 17:14186-14204.

Umesha B, Basavaraju YB. Synthesis and characterization of novel benzo[ $[d][1,3]$ dioxole gathered pyrazole derivatives and their antimicrobial evaluation. Med Chem Res, 2014; 23:3744-3751.

Liu Y-Q, Liu Y, Xiao H, Gao R, Tian X. Synthesis and insecticidal activities of novel derivatives of podophyllotoxin: Part XII. Pest Biochem Physiol. 2008; 9:116-121.

How to cite this article:

Basavaiah U, Sowbhagya, Vinaya, Basavaraju YB. Synthesis and Evaluation of Antimitotic Activity of $N$-Phenyl Tetralones. J App Pharm Sci, 2018; 8(05): 094-100. 